Nature Reviews Gastroenterology \& Hepatology 10, 64 (2013); published online 15 January 2013 ; doi:10.1038/nrgastro.2013.3; doi:10.1038/nrgastro.2013.4; doi:10.1038/nrgastro.2013.5 doi:10.1038/nrgastro.2013.6

\section{IN BRIEF}

\section{COLORECTAL CANCER}

Chinese people in Australia have a lower prevalence of colorectal lesions than white people in Australia

The results of colonoscopies performed at an outpatient practice in Australia have been compared in 346 Chinese and 654 white patients to determine the rates of premalignant polyps. The prevalence of adenomatous polyps was similar in both groups. However, advanced adenomas and sessile serrated adenomas were more common in white patients than in Chinese patients.

Original article Kumbhari, V. et al. Prevalence of adenomas and sessile serrated adenomas in Chinese compared with Caucasians. J. Gastroenterol. Hepatol. doi:10.1111/jgh.12100

\section{PANCREATIC CANCER}

Endoscopic-ultrasonography-guided fine-needle aspiration is effective for diagnosing pancreatic cystic neoplasms

A meta-analysis of 18 studies ( $n=1,438$ patients) has been conducted to determine the accuracy of endoscopicultrasonography-guided fine-needle aspiration (EUS-FNA) for differentiating mucinous from nonmucinous pancreatic cystic lesions. EUS-FNA was found to have moderate sensitivity and high specificity for mucinous lesions. The diagnostic accuracy was greatest in prospective studies and those that lasted $<36$ months. EUS-FNA in combination with cross-sectional imaging could be a useful diagnostic tool for mucinous cysts.

Original article Thornton, G. D. et al. Endoscopic ultrasound guided fine needle aspiration for the diagnosis of pancreatic cystic neoplasms: a meta-analysis. Pancreatology doi:10.1016/j.pan.2012.11.313

\section{BARRETT OESOPHAGUS}

New marker for predicting neoplastic progression in Barrett oesophagus

Researchers have conducted a case-control study to assess the value of using p53 to predict the risk of progression to neoplasia in patients with Barrett oesophagus. The cohort included 720 patients with Barrett oesophagus; those who developed high-grade dysplasia or oesophageal carcinoma were classified as cases and those who did not were classified as controls. Overexpression of p53 was found to be associated with an increased risk of neoplastic progression in these patients. Loss of p53 expression resulted in an even higher risk of progression.

Original article Kastelein, F. et al. Aberrant p53 protein expression is associated with an increased risk of neoplastic progression in patients with Barrett's oesophagus. Gut doi:10.1136/gutjnl-2012-303594

\section{IBD}

\section{New tool for measuring disability in IBD}

An international research team conducted a literature review and canvassed experts to develop a new questionnaire to assess disability in patients with IBD. The new questionnaire was compared with SF-36 and short-IBD in 81 patients. They found that the new questionnaire was able to detect disability and was reliable and reproducible. Long-term testing in large groups of ethnically diverse patients from different countries now needs to be carried out to confirm the findings.

Original article Allen, P. B. et al. Development and validation of a patient-reported disability measurement tool for patients with inflammatory bowel disease. Aliment. Pharmacol. Ther. doi:10.1111/apt.12187 